
СО СДАВЛЕНИЕМ ОРГАНОВ ШЕИ

\author{
() Е.Г. Григорьев ${ }^{1,2 *}$, Е.А. Ильичева ${ }^{1,3}$, Г.А. Берсенев ${ }^{1}$, В.Н. Махутов ${ }^{3}$, Т.Ю. Серебренникова ${ }^{3}$
}

'Иркутский научный центр хирургии и травматологии, Иркутск, Россия

${ }^{2}$ Иркутский государственный медицинский университет, Иркутск, Россия

3 Иркутская ордена «Знак почета» областная клиническая больница, Иркутск, Россия

Представлено клиническое наблюдение хирургического лечения пациента с многоузловым шейно-загрудинным зобом 2 степени по ВО3, осложненным сдавлением трахеи (до 5 мм). Впервые диагноз многоузлового зоба был выставлен эндокринологом по месту жительства 3 года назад. В течение двух лет пациента беспокоили жалобы на затрудненное дыхание и одышку при физической нагрузке. Неоднократно в течение последнего года обращался к специалистам терапевтического профиля (терапевту, пульмонологу, кардиологу), однако ни один из них не смог объяснить причину затрудненного дыхания. После обследования у эндокринного хирурга было выявлено осложненное течение многоузлового зоба с развитием компрессионного синдрома шеи с сужением трахеи. По данным ультразвукового исследования, щитовидная железа диффузно увеличена в размерах, преимущественно за счет левой доли с общим объемом 132,5 см³ по данным компьютерной томографии - трахея смещена вправо за счет компрессии левой долей, просвет сужен до 5 мм. Выставлены показания к оперативному вмешательству по жизненным показаниям. Пациенту выполнена тиреоидэктомия. В послеоперационном периоде жалоб на затрудненное дыхание пациент не предъявлял и отмечал улучшение физической активности. Данное наблюдение показывает, что при ведении пациентов с многоузловым зобом врачу любой специальности необходимо помнить о проявлениях компрессионного синдрома шеи. Следует направлять таких больных как можно раньше на консультацию эндокринного хирурга, чтобы не упустить оптимальные сроки планового оперативного вмешательства.

КЛЮЧЕВЫЕ СЛОВА: щейно-загрудинный многоузловой зоб; сдавление органов шеи; стеноз трахеи; клинический случай; тиреоидэктомия.

\title{
CASE REPORT OF TOXIC MULTINODULAR GOITER WITH COMPRESSION SYNDROME
}

(c) Eugene G. Grigoryev ${ }^{1,2 *}$, Elena A. Ilyicheva ${ }^{1,3}$, Gleb A. Bersenev', Valeriy N. Makhutov³, Tatyana Yu. Serebrennikova ${ }^{3}$

'Irkutsk Scientific Centre of Surgery and Traumatology, Irkutsk, Russia

${ }^{2}$ Irkutsk State Medical University, Irkutsk, Russia

3rkutsk Regional Clinical Hospital, Irkutsk, Russia

We present a case of a 62-year-old patient with multinodular substernal goiter and tracheal compression (up to $5 \mathrm{~mm}$ ). The multinodular goiter was initially diagnosed 3 years before by a local endocrinologist. The patient had been suffering from difficulty of breathing and exertional dyspnea for two years. He consulted specialists in therapy, pulmonology, cardiology more than once. However, none of the clinicians was able to identify the cause of labored respiration. Eventually an endocrine surgeon diagnosed a case of the complicated multinodular goiter with the development of cervical compression syndrome and tracheal narrowing.

Ultrasound study showed the diffuse enlargement of the thyroid gland mainly due to the large left lobe with a total volume of $132,5 \mathrm{~cm}^{3}$. Computed tomography showed the shift of trachea to the right because of its compression by the left lobe and the luminal narrowing up to $5 \mathrm{~mm}$. The patient needed surgical intervention by life-saving indications. He underwent thyroidectomy. The postoperative period was uneventful, the patient did not complain about labored respiration and reported the improvement of physical activity. It is important to keep in mind that patients with multinodular goiter have the potential risk of developing cervical compression syndrome. Current case demonstrates that such patients should be examined by an endocrine surgeon as early as possible in order to perform timely elective surgery.

KEYWORDS: cervico-thoracic multinodular goiter; compression syndrome; tracheal stenosis; comorbidity; case report; thyroidectomy.

\section{АКТУАЛЬНОСТЬ}

Около трети населения мира живет в районах с йодным дефицитом [1]. Распространенность узлового зоба в данных регионах может достигать 80\% [2]. В западной популяции в районах без йодного дефицита узловой зоб выявляется у 6,4\% женщин и 1,5\% мужчин Британии [3] и у 33\% взрослого населения Германии [4]. В нашей стране заболеваемость узловым зобом составляет от 5,2 до 70\% в зависимости от региона [5].

В большинстве случаев зоб имеет шейную локализацию и в 20\% распространяется загрудинно [6]. Особенностью течения шейно-загрудинной локализации является медленный рост зоба, что в большинстве наблюдений 
приводит к поздней обращаемости населения за медицинской помощью $[7,8]$. К моменту обращения у части пациентов определяется узловой/многоузловой $30 б$ больших или гигантских размеров, который приводит к развитию компрессионного синдрома шеи [9]. При загрудинной локализации зоба ткань щитовидной железы (ЩЖ) в 80\% наблюдений распространяется в переднее средостение и около 10-15\% - в заднее [10]. Сдавление органов шеи при доброкачественных заболеваниях диагностируют в 10\%, причем сужение трахеи встречается у 84\% больных этой группы [9, 11, 12].

Актуальность проблемы хирургического лечения зоба, осложненного компрессией трахеи, подтверждается значительным числом отечественных и зарубежных публикаций, связанных с компрессионным синдромом шеи [8-11], в том числе по неотложным показаниям в связи с развитием асфиксии $[9,13]$. Большое количество публикаций посвящено обеспечению анестезиологического пособия у данной группы пациентов [14], а также дооперационной диагностике [15-17].

Проявления компрессии разнообразны: появление «свистящего дыхания» на вдохе/выдохе, одышки в 73,2\% случаев [15], дисфагии - 8,5\% [16], синдромов верхней полой вены и Бернара-Горнера - 3,2\% [17]. Синдром верхней полой вены развивается при распространении ткани ЩЖ загрудинно, что приводит к сдавлению верхней полой вены и нарушению кровообращения в бассейне этого сосуда. Данный синдром включает в себя классическую триаду симптомов: отечность лица, верхней половины туловища и верхних конечностей, цианоз, расширение подкожных вен шеи, верхней половины туловища.

Синдром Бернара-Горнера развивается при зобе крупных и гигантских размеров, который приводит к компрессии преганглионарных волокон симпатических нервов глаза. Проявлениями этого синдрома являются: сужение зрачка (миоз), западение глазного яблока (энофтальм) и снижение ширины глазной щели (псевдоптоз). Менее 1\% случаев сопровождаются кашлем, хронической гипоксией, асфиксией, сдавлением диафрагмального нерва, легочно-сердечной и церебральной недостаточностью [11]. Сдавление органов шеи при доброкачественных узловых заболеваниях ЩЖ является показанием к оперативному вмешательству [18, 19].

Врачами первичного звена проявления компрессионного синдрома нередко интерпретируются как признаки сопутствующей патологии, что влечет за собой длительное безрезультатное медикаментозное лечение, и оптимальные сроки оперативного лечения упускаются [13]. Прогрессирующее сдавление органов шеи, пре- жде всего трахеи, нарастание гипоксии определяют показания к операции по жизненным показаниям.

Ниже приводим клиническое наблюдение.

\section{ОПИСАНИЕ НАБЛЮДЕНИЯ}

Пациент 62 лет поступил в отделение торакальной хирургии ГБУЗ ИОКБ 04.09.2019 г. с жалобами на затрудненное дыхание в покое и одышку при минимальной физической нагрузке. Диагноз многоузлового зоба был установлен в 2017 г. Позднее выявлена декомпенсация функциональной автономии ЩЖ с развитием субклинического тиреотоксикоза. Данные лабораторного обследования представлены в таблице.

Назначена тиреостатическая терапия - тиамазол 20 мг в сутки в течение 4 нед с последующим переходом на 5 мг в сутки. С 2018 г. пациент предъявляет жалобы на одышку, свистящее дыхание. Неоднократно в течение последнего года обращался к специалистам терапевтического профиля (терапевту, пульмонологу, кардиологу), однако ни один из них не смог объяснить причину затрудненного дыхания. В июне 2019 г. уровень ТТГ составил 9,3 мкМЕ/мл, тиамазол был отменен. В августе 2019 г. направлен на консультацию эндокринного хирурга по настоятельному желанию пациента.

\section{Результаты физикального, лабораторного}

и инструментального исследования

Пациент с ожирением II степени - рост 172 см, вес 104 кг, индекс массы тела 35,3 кг/м². Кожа бледно-розовой окраски, подкожная жировая клетчатка чрезмерно развита. При осмотре области шеи: ЩЖ увеличена в размерах за счет обеих долей (2-я степень по ВО3), распространяется загрудинно. При пальпации однородная, мягкая, подвижная, безболезненная. Отмечается смешанная одышка с частотой дыхательных движений до 20 в минуту. В легких дыхание везикулярное, хрипов нет. Тоны сердца приглушены, ритмичные. Число сердечных сокращений 90 в минуту, пульс 90 в минуту. Артериальное давление на обеих плечевых артериях 140/90 мм рт. ст. Результаты лабораторного обследования представлены в таблице 1.

Ультразвуковое исследование (02.06.2020): объем правой доли 82,5 $\mathrm{cm}^{3}$, левой доли - $50 \mathrm{~cm}^{3}$, общий объем - 132,5 см³. По всей паренхиме определяются солидные изоэхогенные узловые образования шаровидной формы с гипоэхогенными контурами размерами максимально справа - 17×10×16 мм, слева - 20×33×40 мм, в области перешейка - 20×10 мм, что соответствует категории TI-RADS 4 в модификации J.Y. Kwak и соавт. (2011).

Таблица. Данные лабораторного обследования

\begin{tabular}{llccc}
\hline \multicolumn{1}{c}{ Показатель } & \multicolumn{1}{c}{$\begin{array}{c}\text { Единицы } \\
\text { изменения }\end{array}$} & $\begin{array}{c}\text { Референсные } \\
\text { значения }\end{array}$ & \multicolumn{2}{c}{ Дата } \\
\hline Тиреотропный гормон (ТТГ) & мкМЕ/мл & $0,4-4,00$ & $\mathbf{0 7 . 0 7 . 2 0 1 7}$ & $\mathbf{0 2 . 0 9 . 2 0 2 0}$ \\
Свободный тироксин (св. Т4) & пмоль/л & $9,00-22,20$ & 0,05 & 3,26 \\
Антитела к тиреопероксидазе & МЕ/мл & $0-34$ & 15,92 & - \\
Антитела к рецепторам ТТГ & МЕ/л & $\leq 1,75$ & 0 & - \\
Кальцитонин & пг/мл & $\leq 9,52$ & 0,16 & - \\
\hline
\end{tabular}


Регионарные лимфатические узлы размерами 6-7 мм, с васкуляризацией, имеются кистозные изменения, гиперэхогенные включения отсутствуют. Наличие узловых образований ЩЖ более 1 см в диаметре явилось показанием к проведению тонкоигольной аспирационной биопсии (ТАБ). Цитологическое заключение пунктатов (10.09.2019) всех узлов ЩЖ диаметром >1см, полученных при ТАБ: узловой коллоидный зоб, что соответствует II диагностической категории по классификации Bethesda (2009). По данным сцинтиграфии ЩЖ (07.08.2017): тиреомегалия, сцинтиграфические признаки повышения функциональной активности тиреоидной ткани в виде множества автономных участков в левой и правой доле ЩЖ, индекс захвата радиофармпрепарата технеций $99 \mathrm{~m}-2,8 \%$, популяционный референс - 1,5-1,9\%, очаговые изменения по типу холодных узлов. Мультиспиральная компьютерная томография (02.09.2020): ЩЖ диффузно увеличена, распространяется загрудинно. Наблюдается смещение трахеи вправо за счет компрессии левой доли, просвет сужен до 5 мм (рис. 1 a, b, c). Эхокардиография: признаки нарушения релаксации миокарда левого желудочка.
Клинический диагноз

Основной. Многоузловой шейно-загрудинный зоб 2-й степени по ВОЗ. Функциональная автономия ЩЖ, диффузная форма, декомпенсация. Тиреотоксикоз субклинический, медикаментозный гипотиреоз. Осложнения основного заболевания. Синдром компрессии органов шеи (сдавление трахеи до 5 мм). Сопутствующие заболевания: гипертоническая болезнь 1-й степени, риск 2; хроническая сердечная недостаточность, 1-й функциональный класс. Учитывая сдавление органов шеи с сужением просвета трахеи до 5 мм, пациенту показано оперативное вмешательство по жизненным показаниям.

В предоперационном периоде осмотрен анестезиологом, выявлено несколько факторов, предрасполагающих к трудностям во время интубации. У пациента имелось ожирение II степени, короткая шея. Рот пациента открывался на 3 см, диапазон движения шеи составил $70^{\circ}$ от полного сгибания до полного разгибания. Класс Маллампати (визуальному осмотру доступно только твердое небо) - IV. Сумма баллов по шкале оценки
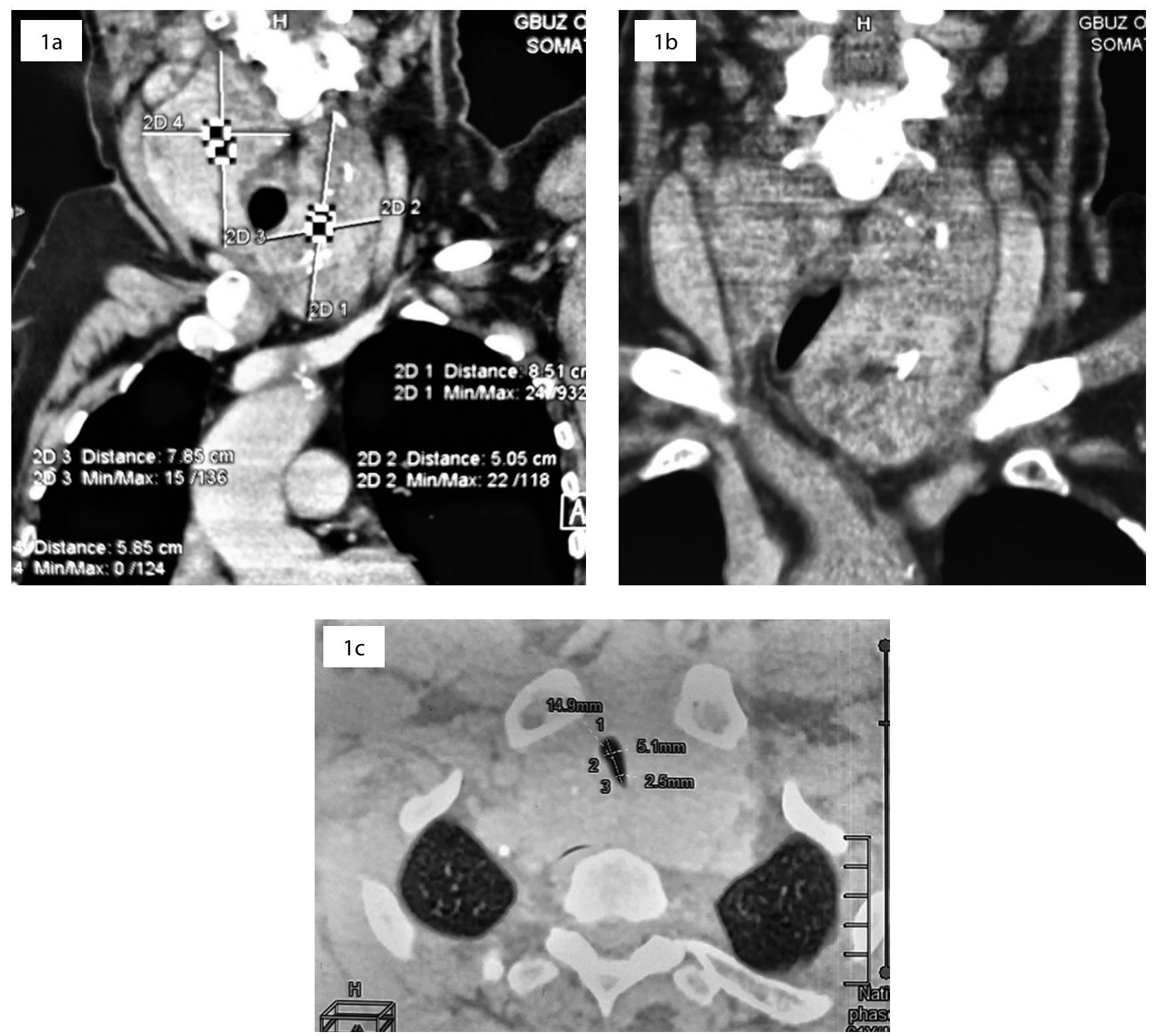

Рисунок 1. Компьютерная томография шеи.

$1 \mathrm{a}$ - сагиттальная проекция; $1 \mathrm{~b}$ - фронтальная проекция; 1с - аксиальная проекция: диффузное увеличение щитовидной железы за счет обеих долей с распространением загрудинно. Верхние полюса достигают сосцевидных отростков. Сужение просвета трахеи до 5 мм. 
трудной интубации ШОТИ составила 4 балла. Запланировано проведение интубации с участием врача-эндоскописта.

Операция состоялась 5.09.2019. г. При поступлении в операционную показатели неинвазивного мониторинга составляли: артериальное давление - 158/90 мм рт.ст., пульс - 80 в минуту, насыщение периферической артериальной крови кислородом $\left(\mathrm{SpO}_{2}\right)-95 \%$ при вдыхании комнатного воздуха. Проводилась преоксигенация 80\% кислородом через кислородную маску. На момент интубации показатели: артериальное давление - 138/78 мм рт.ст., пульс - 72 в минуту, насыщение периферической артериальной крови кислородом $\left(\mathrm{SpO}_{2}\right)$ - 99\%. Первая попытка интубации с прямой ларингоскопией не удалась. Вторая попытка под контролем видеобронхоскопа с использованием армированной интубационной трубки №6 - успешна. Был использован наркозный аппарат Drägerfabius, режим вентиляции - cmv (control mandatory ventilation), минутный объем вентиляции (Ve) - 560 мл, частота вентиляции (f) - 12 л в минуту, пиковое давление выдоха (Ppeak) - 19 см вод.ст., соотношение продолжительности фаз дыхательного цикла (I:P) - 1:2. После подтверждения адекватной вентиляции с помощью капнографии и пульсоксиметрии по стандартной методике выполнены цервикотомия и доступ к ЩЖ. ЩЖ увеличена в размерах, неоднородная, плотной консистенции, верхние полюса достигают сосцевидных отростков височных костей, нижние полюса уходят загрудинно. После пересечения верхних щитовидных артерий и вен, рассечения перешейка произведена экстрафасциальная мобилизация правой и левой долей ЩЖ и пирамидной доли с использованием ультразвукового скальпеля Harmonic с визуализацией и сохранением возвратных нервов, околощитовидных желез в типичном месте. ЩЖ удалена (рис. 2). После удаления железы визуально: деформация трахеи полностью исчезла, хрящевые кольца имеют ровный вид. На момент экстубации с целью уменьшения послеоперационного отека ткани в зоне операции внутривенно введено 90 мг преднизолона в форме 3\% раствора.

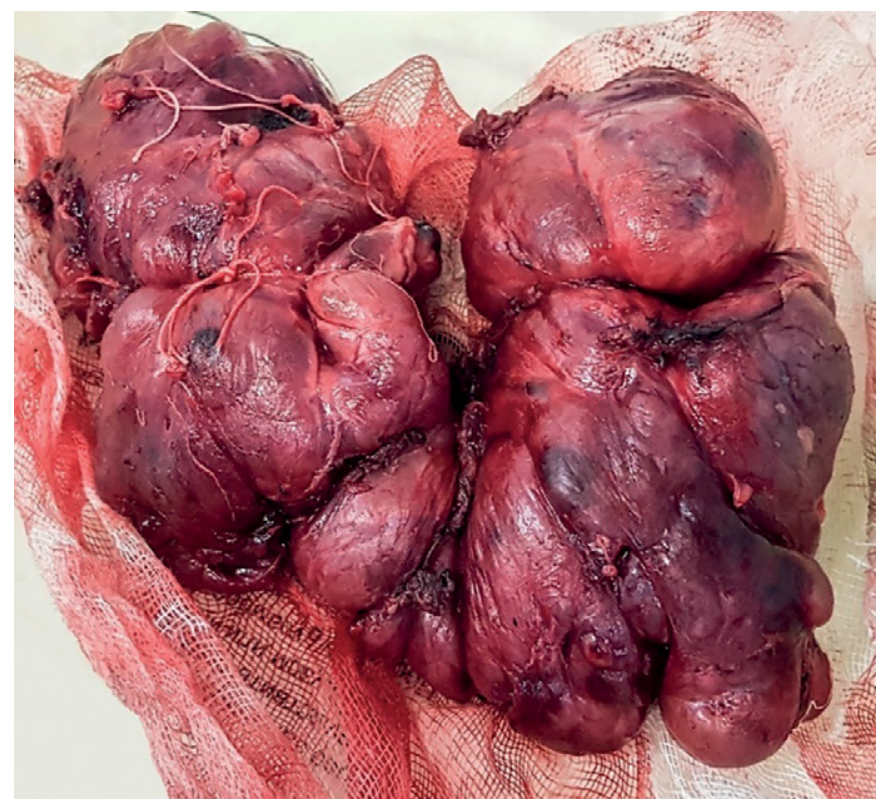

Рисунок 2. Макропрепарат щитовидной железы.
Гистологическое заключение: правая доля ЩЖ $9 \times 9 \times 4 \mathrm{~cm}$, перешеек 4,5×2 cм, левая доля $12 \times 6 \times 4 \mathrm{~cm}$. На разрезе узлы размерами до $3,5 \times 2,5$ см. Гистологическая картина соответствует узловой гиперплазии щЖ. Узлы преимущественно макро- и нормофолликулярного типа строения, с умеренной пролиферацией тиреоидного эпителия с наличием зон резорбции, один из узлов паренхиматозного типа строения. Морфологический диагноз: узловой токсический зоб.

Ранний послеоперационный период протекал без осложнений. Фиброларингоскопия на 1-е сутки - без патологии. Послеоперационной гипокальциемии не было. Швы сняты на 7-е сутки, заживление первичным натяжением. В послеоперационном периоде дыхание свободное. Пациент выписан на 7-е сутки после операции в удовлетворительном состоянии на амбулаторное лечение с назначением L-тироксина 150 мкг 1 р/сут (из расчета 1,6 мкг на 1 кг веса пациента, доза округлена) с последующей коррекцией дозы эндокринологом по уровню ТТГ. Пациент осмотрен спустя 1 и 3 мес после операции - состояние удовлетворительное, жалоб на затруднение дыхания и одышку не предъявляет. Уровень ТТГ через 3 мес составил 1,2 мкME/мл.

\section{ОБСУЖДЕНИЕ}

Заболеваемость доброкачественной патологией щЖ остается высокой как в нашей стране, так и во всем мире в целом $[2,3,5,20]$. Согласно международным и национальным клиническим рекомендациям, основными методами лечения многоузлового токсического зоба являются терапия радиоактивным йодом или тиреоидэктомия $[18,19]$. Назначение кратковременной тиреостатической терапии возможно для достижения эутиреоза перед радикальным лечением. Длительная терапия тиреотоксикоза целесообразна лишь в случае невозможности проведения радикального лечения (престарелый возраст, наличие тяжелой сопутствующей патологии). У части пациентов заболевание осложняется развитием компрессионного синдрома органов шеи [9, 11, 12]. В ряде случаев проявления данного синдрома длительное время не связываются с заболеванием ЩЖ. Возможными причинами этого могут быть: длительный медленный рост ткани ЩЖ с постепенным возникновением симптомов компрессии шеи [7, 8]; низкая информированность о проявлениях компрессионного синдрома врачами первичного звена [13]; принадлежность пациентов к возрастной группе от 50 лет и старше с выраженной коморбидной патологией $[7,11,20]$.

В представленном клиническом наблюдении при установленном диагнозе многоузлового зоба была выбрана неправильная тактика лечения. Назначение тиреостатической терапии без информирования пациента о возможности лечения радиоактивным йодом или тиреоидэктомии было патогенетически необоснованным и нецелесообразным. Кроме того, длительная бесконтрольная терапия тиамазолом привела к ожидаемому нежелательному зобогенному эффекту. Итогом явилось развитие компрессионного синдрома органов шеи, одышка при котором была интерпретирована как следствие избыточного веса. Недооценка выраженности этого симптома привела к задержке рентгенологического обследования и позднему обращению к хирургу. В то же время значительное 
увеличение размеров ЩЖ, выявленное при первичном ультразвуковом исследовании, развитие тиреотоксикоза на фоне многоузлового зоба давало основание поставить вопрос о целесообразности хирургической консультации при первичном обращении пациента.

Особенностью анестезиологического пособия при значительном увеличении размеров ЩЖ и сужении просвета трахеи является трудная интубация [14]. Для преодоления этого риска используются различные технические приемы: использование ларинготрахеальной маски, интубация с помощью видеоларингоскопа или фибробронхоскопа. В нашем случае трудность интубации усугублялась ожирением II степени, короткой шеей, открыванием рта на 3 см, IV классом Маллампати. Использование гибкого эндоскопа позволило обезопасить больного.

Особенностью хирургического вмешательства при значительномувеличении размеров ЩЖявляется затруднение визуализации анатомических образований (возвратно-гортанных нервов, околощитовидных желез), расположенных по латеральной и задней поверхности ЩЖ, значительное ограничение маневра в ране. Рассечение перешейка позволяет увеличить подвижность долей щЖ и уменьшить натяжение тканей при выделении возвратно-гортанных нервов. Отсутствие осложнений в раннем послеоперационном периоде показывает возможность выполнения тиреоидэктомии у подобной категории пациентов.

\section{ЗАКЛЮЧЕНИЕ}

При наблюдении пациентов с многоузловым зобом врачу любой специальности необходимо помнить о проявлениях компрессионного синдрома органов шеи. Представленный клинический случай наглядно демонстрирует, как поздняя диагностика привела к несвоевременно поставленному диагнозу компрессии органов шеи. Лечение сопровождалось трудностями анестезиологического пособия, которые были решены благодаря многолетнему опыту коллектива клиники в работе с данной группой пациентов при плановых операциях. Поэтому следует направлять таких больных как можно раньше на консультацию эндокринного хирурга, чтобы не упустить оптимальные сроки планового оперативного вмешательства.

\section{ДОПОЛНИТЕЛЬНАЯ ИНФОРМАЦИЯ}

Источник финансирования. Исследование и публикация статьи осуществлены на личные средства авторского коллектива.

Согласие пациента. Информированное согласие пациента на публикацию персональной медицинской информации в обезличенной форме было получено.

Конфликт интересов. Авторы декларируют отсутствие явных и потенциальных конфликтов интересов, связанных с публикацией настоящей статьи.

Вклад авторов. Ильичева Е.А., Берсенев Г.А. - разработка концепции и дизайна, анализ и интерпретация данных, обоснование рукописи и проверка критически важного интеллектуального содержания; Григорьев Е.Г. - разработка концепции и дизайна, анализ и интерпретация данных, обоснование рукописи и проверка критически важного интеллектуального содержания, редактирование и окончательное утверждение рукописи; Махутов В.Н., Серебренникова Т.Ю. - сбор материала, анализ и интерпретация данных.

\section{СПИСОК ЛИТЕРАТУРЫ | REFERENCES}

1. Zimmermann MB. lodine Deficiency. Endocr Rev. 2009;30(4):376-408. doi: https://doi.org/10.1210/er.2009-0011

2. Vanderpump MPJ. The epidemiology of thyroid disease. Br Med Bull. 2011;99(1):39-51. doi: https://doi.org/10.1093/bmb/ldr030

3. Vander JB, Gaston EA, Dawber TR. The significance of nontoxic thyroid nodules. Final report of a 15-year study of the incidence of thyroid malignancy. Ann Intern Med. 1968;69(3):537-540. doi: https://doi.org/10.7326/0003-4819-69-3-537

4. Reiners C, Wegscheider K, Schicha H, et al. Prevalence of Thyroid Disorders in the Working Population of Germany: Ultrasonography Screening in 96,278 Unselected Employees. Thyroid.

2004;14(11):926-932. doi: https://doi.org/10.1089/thy.2004.14.926

5. Трошина Е.А., Платонова Н.М., Абдулхабирова Ф.М., Герасимов Г.А. Йододефицитные заболевания в Российской Федерации: время принятия решений / Под ред. И.И. Дедова, Г.А. Мельниченко. М.: Конти-Принт; 2012. - 32 c. [Troshina EA, Platonova NM, Abdulkhabirova FM, Gerasimov GA. Yododefitsitnye zabolevaniyav Rossiyskoy Federatsii: vremya prinyatiya reshenii. Ed.by Dedov II, Melnichenko GA. Moscow: Konti-Print; 2012. 32 p. (In Russ.)].

6. Jennings A. Evaluation of substernal goiters

using computed tomography and $\mathrm{mr}$ imaging.

Endocrinol Metab Clin North Am. 2001;30(2):401-414.

doi: https://doi.org/10.1016/S0889-8529(05)70192-4

7. Березкина И.С., Саприна Т.В., Зима А.П., и др. Клинические особенности и результаты лабораторно-инструментального обследования пациентов с узловыми образованиями щитовидной железы // Клиническая медицина. — 2017. T. 95. — № 4. - C. 355-361. [Berezkina IS, Saprina TV, Zima AP, et al. Clinical features and results of laboratory and instrumental evaluation of patients with nodular formations of the thyroid gland in real clinical practice. Klinicheskaia meditsina. 2017;95(4):355-361. (In Russ.)]. doi: https://doi.org/10.18821/0023-2149-2017-95-4-355-361
8. Lin Y-S, Wu H-Y, Lee C-W, et al. Surgical management of substernal goitres at a tertiary referral centre: A retrospective cohort study of 2,104 patients. Int J Surg. 2016;27(4):46-52. doi: https://doi.org/10.1016/j.jisu.2016.01.032

9. Шулутко А.М., Семиков В.И., Грязнов С.Е., и др. Трудности экстренного хирургического лечения больных зобом с острой дыхательной недостаточностью в результате компрессионного синдрома (клинические наблюдения) // Московский хирургический журнал. - 2015. - №3. - С. 5-11. [Shulutko AM, Semikov VI, Gryaznov SE, et al. Difficulty emergency surgical treatment goiter with acute respiratory failure as a result -compression syndrome (clinical observation). Moskovskii khirurgicheskii zhurnal. 2015;(3):5-11. (In Russ.)].

10. Madjar S, Weissberg D. Retrosternal Goiter. Chest. 1995;108(1):78-82. doi: https://doi.org/10.1378/chest.108.1.78

11. Пиксин И.Н., ВилкОв А.В., Давыдкин В.И., и др. Особенности лечебно-диагностической тактики при компрессионном синдроме у больных с доброкачественными заболеваниями щитовидной железы // Таврический медико-биологический вестник. - 2017. - Т. 20. — № 3-2. - C. 221-226. [Piksin IN, Vilkov AV, Davydkin VI, et al. Peculiarities of diagnostic and treatment tactics when compression syndrome in patients with benign thyroid diseases. Tavricheskii mediko-biologicheskii vestnik. 2017;20(3-2):221-226. (In Russ.)].

12. Chen AY, Bernet VJ, Carty SE, et al. American Thyroid Association Statement on Optimal Surgical Management of Goiter. Thyroid. 2014;24(2):181-189. doi: https://doi.org/10.1089/thy.2013.0291

13. Щеголев А.А., Ларин А.А., Хитрова Е.Н., и др. Неотложная резекция щитовидной железы у 80-летней пациентки с загрудинным зобом и компрессионным синдромом // Клиническая геронтология. 2010. - T. 16. — № 7-8. - C. 64-65. [Shchegolev AA, Larin AA, Khitrova EN, et al. Emergency resection of the thyroid gland in 80-year-old patient with retrosternal goiter and compression syndrome. Klinicheskaia gerontologiia. 2010;16(7-8):64-65. (In Russ.)]. 
14. Неговский А.А., Шпажникова Т.И., Знаменский А.А., и др. Анестезиологическое обеспечение операций на щитовидной железе // Общая реаниматология. - 2008. - Т. 4. - № 6. - С. 65. [Negovsky AA, Shpazhnikova TI, Znamensky AA, et al. Anesthetic maintenance of thyroid surgery. General Reanimatology. 2008;4(6):65 (In Russ.)]. doi: https://doi.org/10.15360/1813-9779-2008-6-65

15. Sadrizadeh A, Ghafarian S, Haghi SZ, Salehi M. Evaluations of factors predicting the need for an extra-cervical approach for intra-thoracic goiter. Iran J Otorhinolaryngol. 2015;27(83):435-441. doi: https://doi.org/10.22038/ijorl.2015.5401

16. Nistor C, Ciuche A, Motaş C, et al. Cervico-mediastinal thyroid masses - our experience. Chirurgia (Bucur). 2014;109(1):34-43.

17. Giulea C. Pemberton's Sign and Intense Facial Edema in Superior Vena Cava Syndrome due to Retrosternal Goiter. Acta Endocrinol. 2016;12(2):227-229. doi: https://doi.org/10.4183/aeb.2016.227

18. Бельцевич Д.Г., Ванушко В.Э., Мельниченко Г.А., и др. Клинические рекомендации Российской ассоциации эндокринологов по диагностике и лечению (много) узлового зоба у взрослых // Эндокринная хирургия. 2016. - T.10. - №1. - C. 5-12. [Beltsevich DG, Vanushko VE, Melnichenko GA, et al. Russian Association of Endocrinologists Clinic Guidelines for thyroid nodules diagnostic and treatment. Endocrine Surgery. 2016;10(1):5-12. (In Russ.)] doi:https://doi.org/ 10.14341/serg201615-12

19. Ross DS, Burch HB, Cooper DS, et al. 2016 American Thyroid Association Guidelines for Diagnosis and Management of Hyperthyroidism and Other Causes of Thyrotoxicosis. Thyroid. 2016;26(10):1343-1421. doi: https://doi.org/10.1089/thy.2016.0229

20. Аметов А.С., Кондратьева Л.В., Бугова Л.А. Болезнь Грейвса и функциональная автономия щитовидной железы в регионе с легким йодным дефицитом // Клиническая и экспериментальная тиреоидология. — 2011. - Т. 7. — № 4. C. 51-55. [Ametov AS, Kondratieva LV, Bugova LA. Graves' disease and thyroid functional autonomy in the region of mild iodine deficiency. Clinical and experimental thyroidology.2011;7(4):51-55 (InRuss.)].doi: https://doi.org/10.14341/ket20117451-55

\section{ИНФОРМАЦИЯ ОБ АВТОРАХ [AUTHORS INFO]}

*Григорьев Евгений Георгиевич, д.м.н., профессор, член-корреспондент PAH [Eugene G. Grigoryev, MD, PhD, Professor]. Россия, 664049, г. Иркутск, Юбилейный мкрн, д. 100 [100, Yubileyny microrayon, 664049, Irkutsk, Russian Federation]. ORCID: 0000-0002-5082-7028; SPIN: 8969-4112; e-mail: egg@iokb.ru

Ильичева Елена Алексеевна, д.м.н., професcop [Elena A. llyicheva, MD, PhD, Professor]; ORCID: 0000-0002-2081-8665; SPIN: 3624-4643; e-mail: lena_isi@mail.ru

Берсенев Глеб Александрович, аспирант, врач-хирург [Gleb A. Bersenev]; ORCID: 0000-0002-6887-8325;

SPIN: 1467-8503; e-mail: glbersenev17@gmail.com

Махутов Валерий Николаевич, к.М.Н., заведующий торакальным хирургическим отделением ГБУЗ ИОКБ

[Valeriy N. Makhutov, PhD]; ORCID: 0000-0001-7318-7193; SPIN: 7627-5484; e-mail: iokb@iokb.ru

Серебренникова Татьяна Юрьевна, врач анестезиолог-реаниматолог отделения анестезиологии и реанимации № 1 ГБУЗ ИОКБ [Tatyana Yu. Serebrennikova] ORCID: 0000-0002-2807-4736; SPIN: 1569-8645; e-mail: serebty@yandex.ru

\section{ИНФОРМАЦИЯ}

Рукопись получена: 09.06.2020. Одобрена к публикации: 20.08.2020.

\section{ЦИТИРОВАТЬ:}

Григорьев Е.Г., Ильичева Е.А., Берсенев Г.А., Махутов В.Н., Серебренникова Т.Ю. Клиническое наблюдение токсического многоузлового зоба со сдавлением органов шеи // Эндокринная хирургия. — 2020. — Т. 14. — №2. — С. 10-15. doi: https://doi.org/10.14341/serg12477

\section{TO CITE THIS ARTICLE:}

Grigoryev EG, llyicheva EA, Bersenev GA, Makhutov VN, Serebrennikova TY. Case report of toxic multinodular goiter with neck compression. Endocrine surgery. 2020;14(2): 10-15. doi: https://doi.org/10.14341/serg12477 